\title{
How Misinformation that Facemasks are Effective for Reducing COVID-19 is Transmitted
}

\author{
Colin A. Ross* \\ Institute for Psychological Trauma, 1701 Gateway, Suite 349, Richardson, TX 75080, USA
}

*Corresponding author: Colin A. Ross, Institute for Psychological Trauma, 1701 Gateway, Suite 349, Richardson, TX 75080, USA

Received: August 25, 2020; Accepted: September 07, 2020; Published: September 12, 2020

The evidence from randomized controlled trials (RCTs) that surgical facemasks and cloth facemasks are ineffective for preventing transmission of respiratory viruses in public is conclusive [1-8]. In all the meta-analyses of all the existing RCTs [4-7], not a single trial was found in which facemasks provided any protection against virus transmission in public $[1,2]$. Consistent with the findings of all RCTs comparing transmission rates in public with and without facemasks, Leung et al. compared the rates of detection of three types of viruses in exhalations by infected individuals with and without facemasks: they concluded that, with facemasks, there was: "no significant reduction in detection of influenza virus in aerosols;" "For rhinovirus there were no significant differences between detection of virus with or without facemasks, both in respiratory droplets and in aerosols;" and, for coronavirus there was "no significant reduction in detection in aerosols [3]." They did observe a reduction in detection of viruses in respiratory droplets for influenza virus and coronavirus. There is no doubt that facemasks can reduce the transmission of droplets, but droplets are not the concern for transmission of viruses in public. Significant numbers of droplets are not exhaled by asymptomatic carriers because they are not coughing or sneezing in public. Symptomatic carriers who are coughing and sneezing should be quarantined. The rationale for both recommended and mandated facemasks in public is to reduce transmission by asymptomatic carriers. Since all the RCTs in the literature show no reduction in transmission of viruses in public due to facemasks, one wonders why the CDC, NIH and virtually all medical authorities are stating that there is conclusive scientific evidence that facemasks reduce viral transmission in public.

For example, Brooks, Butler and Redfield [9] stated in the July 14, 2020 issue of JAMA that, "At this critical juncture when COVID-19 is resurging, broad adoption of cloth face covering is a civic duty." Redfield is the Director of the CDC. Similarly, Gostin, Cohen and Koplan [10] stated in the August 11, 2020 issue of JAMA that, "The ethical justification for face coverings is their utility in preventing transmission of serious disease to community members." Gostin et al. recommend delivering a public health message of, "When we all mask up, we are all safer [10]." The scientific facts are that facemasks have no effect on the transmission of viruses in public. This is proven by multiple RCTs. And yet, the CDC, writers in JAMA, and many medical authorities, state that it is scientifically proven that facemasks will protect the public from COVID-19. What is the evidence these authorities cite as scientific proof, while ignoring and never citing the RCTs?

Brooks et al. have 14 references that provide their evidence [9]. Their first reference is a study in health care settings that provides no data on transmission in public. The second and third references are to the Massachusetts Department of Public Health website and provide no data. The fourth paper describes a web app for screening healthcare workers for COVID-19. The fifth reference, published in 2019, provides guidance for how to respond to pandemics. The sixth reference says that asymptomatic carriers can transmit the virus to others but does not discuss or provide any data on facemasks. The seventh reference says that undocumented coronavirus infections can result in transmission to others but does not discuss or provide evidence on facemasks. The eighth reference is to a 1994 physics paper that describes reduction of "respiratory jets" by facemasks but provides no evidence on viral transmission. The ninth reference discusses the filtration efficiency of facemasks but provides no data on transmission in public. The tenth reference discusses the "potential utilities" of hand washing and facemasks but provides no evidence that they work. The eleventh reference is a paper by Greenlagh et al. that provides the authors' responses to their critics but does not provide data on transmission in public [8]. The twelfth reference describes factors affecting whether people use facemasks but no data or evidence on whether facemasks work to reduce transmission in public. The thirteenth paper describes a single situation in which two COVID-positive hair stylists wore masks at work and none of their 104 clients followed up two weeks later reported symptoms of COVID-19; 102 of the 104 clients wore facemasks themselves while at the salon. None of these 104 individuals were tested for COVID-19 and many of them could have been asymptomatic carriers. The fourteenth paper is a discussion of facemasks and Gross National Product in a Goldman Sachs Research publication.

Thus, of Brooks et al's fourteen references, none provide any controlled data on the effects of facemasks on transmission of viruses in public [9]. Gostin et al. provide ten references to support their assertion that it is a civic duty to wear facemasks during the COVID-19 pandemic [10]. The first reference is to a CDC website statement that facemasks work in public and should be worn in public [11]. The CDC website provides 19 references to support their recommendation, but those references consist of small or single case 
uncontrolled studies, discussions of asymptomatic transmission that provide no data on facemasks, papers about infections in residents of long-term care facilities, and papers about the filtration properties of facemasks. None of the CDC references provide any evidence or data that facemasks work in public to reduce the rate of viral transmission.

Returning to the Gostin et al. [10] paper, their second reference is Brooks et al. [9]. Their third reference is to a study that provides no data on reduction of viral transmission rates due to wearing facemasks in public. Their fourth reference is to a study of health care workers not of transmission in public. Their fifth reference is to a study by Lyu et al. [11] that has been previously discussed by Ross and will be discussed below [2]. Their sixth, seventh and eighth references are to single legal cases. Their ninth reference is to a 2007 book by the Institute of Medicine. Their tenth reference discusses the administrate duties of the Social Security Commissioner.

Thus, the CDC website, Brooks et al. and Gostin et al. provide a total of 43 references, none of which provide any controlled evidence that facemasks worn in public reduce the rate of coronavirus transmission $[9,10]$. None of these three authorities reference a single one of the RCTs, all of which found zero evidence that facemasks reduce viral transmission when worn in public. The authorities in these three examples (the CDC, Brooks et al, and Gostin, et al.), and authorities throughout the United States are virtually unanimous in saying that facemasks work, that this has been proven scientifically, and that the public should wear them. This is put forward as a recommendation at the least, and often as a mandate.

The only paper out of the 43 that provides any data on facemasks in public is a study by Lyu et al. [12]. These authors tracked the rates of COVID-19 infections in 15 states and D.C. from March 31 to May 22, 2020. They found that infection rates declined more in the states that instituted mask wearing in public compared to states that did not. The difference in rates varied from $0.9 \%$ to $2.0 \%$. If we assume that during this time period $5 \%$ of the population became infected, this would mean that facemasks could have reduced the number of infections in the population by a maximum of $(0.05 \times 0.02=0.001) 0.1 \%$. However, during that time period the COVID-19 curve was flattening in most of the country. More importantly, multiple variables were contributing to the rate of infections including social distancing, hand washing, quarantines, and business and school closures, so facemasks likely contributed only a fraction of $0.1 \%$, which is not clinically meaningful and is well within the margin of error for viral disease epidemiology in the United States. The CDC's estimate of the number of flu deaths in the United States in a given year is usually stated as a being within a range of tens of thousands of cases. A fluctuation of $0.1 \%$ in the infection rate when the best CDC estimate for annual flu deaths is +/$10,000-20,000$ is completely meaningless.

If single cases and small uncontrolled observational studies with weak methodology were cited to prove the effectiveness of an alternative treatment for pneumonia, a paper reporting those results would never get published in any mainstream journal; the claim that the alternative treatment was effective would be rejected by medical authorities. Yet this is the quality of evidence cited by the CDC, medical authorities and leading medical journals that facemasks are effective for reducing coronavirus transmission in public. That claim has been disproven by four meta-analyses of RCTs. In any case, there is no reason to expect surgical facemasks to work because their pore size is in the range of 50-100 microns, while the coronavirus is about 0.1 microns and aerosols are about 2-3 microns in size [1,2]. The available science shows conclusively that public facemasks don't work. This is not a conspiracy theory, an anti-masker ideological statement, or an anti-medical or anti-authority statement. It is a statement of scientific fact. Facemasks may make people feel safer, and may confer a sense of solidarity, but this is just a feeling. Medically and scientifically, facemasks do not protect the public during the COVID-19 pandemic.

Ignoring these facts will not change them. It is not possible to create confidence in organized medicine by mandating public policies that are proven to be ineffective by medical science. The medical profession is putting itself at risk for blowback by endorsing and mandating public wearing of facemasks. Once statements that facemasks work are made enough times by medical authorities, they become common knowledge, and are transmitted throughout the culture. Hopefully, at some point science, data and sound medicine will prevail. The statement that public wearing of facemasks is ineffective for reducing coronavirus infection rates has been made twice by the same group from Harvard in recent issues of the New England Journal of Medicine: "We know that wearing a mask outside health care facilities offers little, if any, protection from infection [13,14]." In conclusion, it should be noted that the author's mother is in her nineties and living in a long-term care facility. The author is fully supportive of all the COVID-19 precautions instituted at this facility including facemasks, social distancing, quarantining and restriction of visitors during the height of the pandemic and rigorous daily screening of employees. The author is also fully in support of wearing facemasks inside operating rooms. The evidence just does not support wearing facemasks in public.

\section{References}

1. Ross CA (2020) Thoughts on COVID-19. Journal of Neurology and Neurocritical Care 3: 1-3.

2. Ross CA (2020) Facemasks are not effective for preventing transmission of the coronavirus. Journal of Neurology and Neurocritical Care 3: 1-2.

3. Leung NHL, Chu DKW, Cowling BY (2020) Respiratory virus shedding in exhaled breath and efficacy of facemasks. Nature Medicine 26: 676-680.

4. Brainard JS, Jones N, Lake I, Hooper L, Hunter P (2020) Face masks and similar barriers to prevent respiratory illness such as COVID-19: A rapid systematic review. Medrxiv. doi:10.1101/2020.04.01.20049528.

5. Cowling BJ, Zhou Y, Ip DK, Leung GM, Aiello AE (2010) Face masks to prevent transmission of influenza virus: a systematic review. Epidemiology of Infections 138: 449-456.

6. Xiao J, Shiv EYC, Gao H, Wong JY, Fong MW, Ryu S, et al. (2020) Nonpharmaceutical measures for pandemic influenza in nonhealthcare settings - personal protective and environmental measures. Emerging Infectious Diseases 26: 967-975.

7. Aggarwhal N, Dwarakananthan V, Gautham N, Ray A (2020) Facemasks for prevention of viral respiratory infections in community settings: A systematic review and meta-analysis. Indian Journal of Public Health64: 192-200.

8. Greenhalgh T, Schmid MB, Czypionka T, Bassler D, Gruer L (2020) Facemasks for the public during the COVID-19 crisis. British Medical Journal. doi:10.10.1136/bmj. m1435. 
Colin A. Ross (2020) How Misinformation that Facemasks are Effective for Reducing COVID-19 is Transmitted

9. Brooks JT, Butler JC, Redfield RR (2020) Universal masking to prevent SARS-CoV-2 transmission - the time is now. JAMA. doi.10.1001/jama.2020.13107.

10. Gostin LO, Cohen IG, Koplan JP (2020) Universal masking in the United States. The role of mandates, health education and the CDC. JAMA doi.10.1001/ jma.2020.15271.

11. Centers for Disease Control and Prevention (2020) https://www.cdc.gov/coronavirus/ 2019-ncov/prevent-getting-sick/cloth-face-cover- guidance.html.
12. Lyu W, Wehby GL (2020) Community use of face masks and COVID-19: Evidence from a natural experiment of state mandates in the US. Health Affairs. doi. org/10.1337/hlthaff.2020.00828.

13. Klompas M, Morris CA, Sinclair J, Pearson M, Shenoy ES (2020) universal masking in hospitals in the COVID-19 era. New England Journal of Medicine 382: e62-63.

14. Klompas M, Morris CA, Shenoy ES (2020) Universal masking in the COVID-19 era. New England Journal of Medicine 383: e9. 